\title{
Squamous cell carcinoma antigen expression in tumor cells is associated with the chemosensitivity and survival of patients with cervical cancer receiving docetaxel-carboplatin-based neoadjuvant chemotherapy
}

\author{
PENG CHEN $^{1}$, LIANG JIAO ${ }^{2}$ and DAN-BO WANG ${ }^{1}$ \\ ${ }^{1}$ Department of Obstetrics and Gynecology, Shengjing Hospital of China Medical University, Shenyang, Liaoning 110004; \\ ${ }^{2}$ Department of Gynecology, Cancer Hospital of China Medical University, Shenyang, Liaoning 110042, P.R. China
}

Received February 27, 2016; Accepted November 11, 2016

DOI: $10.3892 / \mathrm{ol} .2017 .5560$

\begin{abstract}
The present study examined the association between squamous cell carcinoma antigen (SCCA) expression and the response of patients with cervical cancer to docetaxel-carboplatin (DC) combination chemotherapy, in order to determine the prognostic potential of SCCA expression. A total of 21 patients were enrolled with stage IB2 or stage IIA2 SCC. Of these, 9 patients had chemotherapy-sensitive cancer ( 2 cases with a complete response and 7 cases with a partial response) and 12 patients had chemotherapy-resistant cancer (12 cases of stable disease and no cases of progressive disease). Patients were treated with two cycles of DC chemotherapy (docetaxel $75 \mathrm{mg} / \mathrm{m}^{2}$ plus cisplatin $25 \mathrm{mg} / \mathrm{m}^{2}$ ) with 21-day intervals, followed by radical surgery. SCCA expression levels prior to and following chemotherapy were evaluated using immunohistochemistry. Following DC chemotherapy, the SCCA expression levels decreased in the patients with chemotherapy-sensitive cancer, but not in those with chemotherapy-resistant cancer $(\mathrm{P}=0.042)$. Significant survival differences between the SCCA-positive and -negative patients following chemotherapy $(\mathrm{P}=0.009)$ was observed. However, no statistically significant difference in survival between patients with chemotherapy-sensitive and chemotherapy-resistant cancer, or between patients with SCCA-positive and -negative expression prior to chemotherapy was observed. Overall, the chemotherapy sensitivity of patients with cervical cancer was associated with decreased SCCA expression levels following DC chemotherapy. Therefore, SCCA expression levels following DC chemotherapy may potentially be used in the
\end{abstract}

Correspondence to: Professor Dan-Bo Wang, Department of Obstetrics and Gynecology, Shengjing Hospital of China Medical University, 36 Sanhao Street, Shenyang, Liaoning 110004, P.R. China E-mail: wangdbsj@gmail.com

Key words: cervical cancer, docetaxel-carboplatin combination chemotherapy, squamous cell carcinoma antigen, chemotherapy sensitivity, survival clinical prognosis for cervical cancer patients who receive DC chemotherapy and subsequent radical surgery.

\section{Introduction}

Neoadjuvant chemotherapy (NAC), which is currently the standard approach for patients with locally advanced breast cancer (1), is increasingly used to treat patients with large operable and locally advanced cancer types, including gastric (2), bladder (3), esophageal (4) and cervical $(5,6)$ cancer. At present, cervical cancer is the second most frequent malignant neoplasm, with a poor prognosis among women (7-9). Although various NAC strategies have been developed to increase survival times, the efficacy of this treatment for cervical cancer remains subject to debate, which may be due to the poor response to chemotherapy (chemosensitivity) observed in certain patients $(10,11)$. Therefore, it is necessary to investigate the factors associated with chemosensitivity and survival of patients with cervical cancer.

NAC followed by radical surgery has emerged as an alternative for locally advanced cervical carcinoma therapy from the 1980s onwards (12). Platinum-based chemotherapies have significantly improved the outcomes of cervical cancer treatments $(13,14)$, with cisplatin and carboplatin being the most commonly used within this class of chemotherapeutic agents. Lorusso et al (15) suggested that carboplatin plus paclitaxel-based chemotherapy is equally effective and less toxic compared with cisplatin plus paclitaxel-based chemotherapy. In addition, it was reported that docetaxel was four times more potent than paclitaxel with regard to antiangiogenic activity (16), and had a high efficacy in paclitaxel-resistant cancer types $(17,18)$. Furthermore, the docetaxel-carboplatin (DC) combination chemotherapy was applied for the treatment of advanced-stage cervical cancer and was demonstrated to be well tolerated, with minimal toxic effects (19). Therefore, DC chemotherapy was selected for the treatment of patients with cervical cancer in the current study. A study investigating the markers specifically relevant to the chemosensitivity and survival in patients with cervical cancer receiving DC chemotherapy, at present, has not been performed. Therefore, these potential markers were investigated in the present study. 
Squamous cell carcinoma antigen (SCCA) expression is an established prognostic and predictive factor for cervical cancer (20-22), and a sensitive and reliable indicator for the response of this disease to paclitaxel and carboplatin-based chemotherapy (23). Elevated SCCA expression levels prior to cisplatin-based NAC are associated with a poor response to this therapy (22). Therefore, it was hypothesized that the SCCA protein levels may be associated with the chemosensitivity of patients with cervical cancer to DC chemotherapy. As the heterogeneity of patient chemosensitivity to NAC is primarily determined by factors inherent to the individual and is closely aligned with the clinical response to anticancer drugs (24), the association between SCCA expression and patient chemosensitivity to DC chemotherapy was assessed in the present longitudinal study. Furthermore, the prognostic potential of SCCA expression for predicting the survival of patients with cervical cancer, who received DC chemotherapy followed by radical surgery, was also evaluated.

\section{Patients and methods}

Patients. Between March 2009 and May 2013, 21 patients diagnosed with squamous cervical cancer by biopsy histopathology at the Department of Obstetrics and Gynecology, Shengjing Hospital Affiliated with China Medical University (Shenyang, China) were prospectively enrolled in the current study. The inclusion criteria were as follows: Diagnosis of SCC; stage IB2 or stage IIA2 cancer according to the criteria of The International Federation of Gynecology and Obstetrics (FIGO) stage (25); the patient satisfied the indications for NAC. Patients with the following conditions were excluded: Those unable to undergo surgery due to other diseases; the presence of other types of cancer that may influence SCCA expression levels; contraindications to chemotherapy or radical surgery; the tumor was detected by differing imaging techniques prior to and during treatment. All patients gave written informed consent and the study protocol was approved by the Ethics Committee of Shenjing Hospital.

Chemotherapy. Two cycles of DC chemotherapy (docetaxel $75 \mathrm{mg} / \mathrm{m}^{2}$ by intravenous infusion for $1 \mathrm{~h}$ on day 1 , and cisplatin $25 \mathrm{mg} / \mathrm{m}^{2}$ by infusion for 1-3 h on day 1-3) were performed at 21-day intervals prior to surgery (19). Further radiotherapy or chemotherapy was dependent on the histological results following radical surgery. The maximum diameter of the lesions prior to and following chemotherapy was detected by computed tomography (CT) and magnetic resonance (MR) imaging methods. The sensitivity of the patients with cervical cancer to DC chemotherapy was determined based on the Response Evaluation Criteria in Solid Tumors (RECIST), version 1.0 (26). A total of four response categories for cervical cancer were defined as follows: Complete response (CR; all lesions of cervical cancer disappeared completely and a short axis of lymph nodes, $<10 \mathrm{~mm}$, following two cycles of chemotherapy); partial response [PR; $\geq 30 \%$ decrease in the sum of the longest diameters (SLD) of the lesions]; progressive disease (PD; $\geq 20 \%$ increase in the SLD or an increase in the absolute length of $>5 \mathrm{~mm}$ ); and stable disease (SD; neither CR nor PD). Cervical cancer response categories were assigned by two radiologists. Chemotherapy-sensitive cancer (CR or PR) and chemotherapy-resistant cancer (PD or SD) were determined based on the aforementioned cervical cancer response categories.

Radical surgery (radical hysterectomy or pelvic lymphadenectomy) was performed following chemotherapy. Post-surgery mortality and survival rates were recorded during the follow-up period (until December 2014). The tumor and lymph node tissues were collected 7-10 days prior to chemotherapy and 22-25 days post-chemotherapy, and were subsequently fixed in $10 \%$ neutral-buffered formalin for $24 \mathrm{~h}$ at room temperature and embedded in paraffin.

Immunohistochemistry analysis. The paraffin-embedded tissues were cut at a thickness of $5 \mu \mathrm{m}$ using a microtome (Leica Microsystems, K.K., Tokyo, Japan). Immunohistochemistry analyses were performed according to the manufacturer's protocol (Wuhan Boster Biological Technology, Ltd., Wuhan, China). Briefly, the tissue sections were deparaffinized and rehydrated through a graded ethanol series. Following this, antigen retrieval was conducted by soaking and heating the sections in citric acid buffer $(0.01 \mathrm{M})$ at $100^{\circ} \mathrm{C}$ for $5 \mathrm{~min}$. Hydrogen peroxide solution (1\%) was used to block the activity of endogenous peroxidase for $30 \mathrm{~min}$ at room temperature. The tissue sections were then blocked with 5\% normal goat serum (Sigma-Aldrich; Merck Millipore, Darmstadt, Germany) for $30 \mathrm{~min}$ at $37^{\circ} \mathrm{C}$ to eliminate non-specific binding. Following this, the tissue sections were incubated with a primary rabbit polyclonal antibody against SCCA (no. sc-25499; dilution, 1:200; Santa Cruz Biotechnology, Inc., Dallas, TX, USA) at $4^{\circ} \mathrm{C}$ overnight. The tissue sections were washed with phosphate-buffered saline and incubated with a horseradish peroxidase (HRP)-conjugated secondary antibody (goat anti-rabbit, Wuhan Boster Biological Technology, Ltd.) at room temperature for $1 \mathrm{~h}$, followed by staining using an avidin-biotin-HRP kit (Wuhan Boster Biological Technology, Ltd.) for $30 \mathrm{~min}$ at $37^{\circ} \mathrm{C}$. The tissue sections were subject to a double-blind evaluation by two pathologists.

The brown granules present in the cytoplasm of cells were considered as having positive SCCA expression. Tissue sections with $0,0-10,10-50$ and $>50 \%$ of cells identified as positive for SCCA expression were scored as 0, 1, 2 and 3, respectively (a). Staining intensity was scored as follows: no staining, faint yellow, yellow and brownish-yellow were scored as $0,1,2$ and 3, respectively (b). Immunoreactions were visualized using a light microscope (Axiovert 200M; Zeiss GmbH, Jena, Germany) and Zeiss AxioVision 4.7 software (Carl Zeiss AG, Oberkochen, Germany). The expression intensity of SCCA was determined by multiplying (a) and (b) values, with a total score of 0 defined as SCCA-negative (-) expression and scores of 1-9 defined as SCCA-positive (+) expression (20).

Statistical analysis. SPSS 19.0 software (IBM SPSS, Armonk, NY, USA) was used for statistical analysis. Categorical data were summarized as frequencies and percentages. Continuous data are presented as the mean \pm standard deviation. A $\chi^{2}$ test or Fisher's exact test was used for categorical data analysis, while the Mann-Whitney test was used for continuous data. Overall survival analysis was performed using the Kaplan-Meier method and differences in the survival rates were assessed by 
Table I. Basic characteristics of patients with chemotherapy-sensitive ( 2 complete response and 7 partial response) and chemotherapy-resistant (12 stable disease and 0 progressive disease) cervical cancer.

\begin{tabular}{|c|c|c|c|}
\hline Categories & $\begin{array}{c}\text { Chemotherapy- } \\
\text { sensitive }\end{array}$ & $\begin{array}{l}\text { Chemotherapy- } \\
\text { resistant }\end{array}$ & P-value \\
\hline Positive lymph node number FIGO stage, n (\%) & $0.8 \pm 1.1$ & $1.4 \pm 2.2$ & 0.670 \\
\hline IB2 & $4(44.4)$ & $5(41.7)$ & 1.000 \\
\hline IIA2 & $5(55.6)$ & $7(58.3)$ & \\
\hline Differentiation grades, $\mathrm{n}(\%)$ & & & 0.155 \\
\hline G1 & $4(44.4)$ & $2(16.7)$ & \\
\hline $\mathrm{G} 2$ & $3(33.3)$ & $9(75.0)$ & \\
\hline G3 & $2(22.2)$ & $1(8.3)$ & \\
\hline Infiltration depth, n (\%) & & & 0.397 \\
\hline$<50 \%$ & $4(44.4)$ & $3(25.0)$ & \\
\hline$\geq 50 \%$ & $5(55.6)$ & $9(75.0)$ & \\
\hline Maximum diameter of lesion pre-chemotherapy, $\mathrm{mm}$ & $6.0 \pm 1.0$ & $4.9 \pm 1.7$ & 0.088 \\
\hline Percentage reduction in diameter of lesion post-chemotherapy, $\%$ & $51.2 \pm 22.5$ & $5.3 \pm 22.4$ & $<0.001^{\mathrm{a}}$ \\
\hline
\end{tabular}

${ }^{\text {a }}<0$ 0.05. FIGO, International Federation of Gynecology and Obstetrics.

Table II. Expression of SCCA protein pre- and post-docetaxel-carboplatin chemotherapy in chemotherapy-sensitive (2 complete response and 7 partial response) and chemotherapy-resistant (12 stable disease and 0 progressive disease) cervical cancer patients .

\begin{tabular}{lcc}
\hline Categories & Chemotherapy-sensitive & Chemotherapy-resistant \\
\hline Positive expression rate of SCCA protein & & $10 / 12(83.3 \%)$ \\
Pre-chemotherapy & $7 / 9(77.8 \%)$ & $8 / 12(66.7 \%)$ \\
Post-chemotherapy & $3 / 9(33.3 \%)$ & 0.640 \\
P-value & 0.153 & 0.198 \\
Score for expression intensity of SCCA & & $4.8 \pm 2.7$ \\
Pre-chemotherapy & $4.7 \pm 3.4$ & $2.8 \pm 2.4$ \\
Post-chemotherapy & $1.0 \pm 1.6$ & 0.054 \\
P-value & 0.042 & 0.970 \\
\hline
\end{tabular}

SCCA, squamous cell carcinoma antigen.

the log-rank test. $\mathrm{P}<0.05$ was considered to indicate a statistically significant difference.

\section{Results}

Clinical characteristics of the study subjects. A total of 21 patients with cervical cancer were included in the present study. Patient ages ranged from 26-63 years, with a mean age of 49 years. Based on the RECIST 1.0 criteria, 9 cases were chemotherapy-sensitive ( 2 cases of CR; 7 cases of PR) and 12 cases were chemotherapy-resistant ( 0 cases of PD; 12 cases of SD). The positive lymph node number, FIGO stage, differentiation grade, infiltration depth and the maximum lesion diameter prior to AC of patients with chemotherapy-sensitive and chemotherapy-resistant cancer were similar (all $\mathrm{P}>0.05$ ). As expected, the percentage reduction in the diameter of the lesions of the chemotherapy-sensitive patients was significantly higher following NAC compared with that of patients with chemotherapy-resistant cancer $(\mathrm{P}<0.0001$; Table I).

SCCA expression in cervical cancer. Following treatment with DC chemotherapy, the proportion of cases with SCCA-positive expression had not significantly decreased, but the score for the SCCA expression intensity was significantly reduced post-DC chemotherapy in patients with chemotherapy-sensitive cancer compared with the intensity pre-DC chemotherapy $(\mathrm{P}=0.042$; Table II $)$. However, no significant decrease in SCCA expression intensity was observed post-DC chemotherapy in chemotherapy-resistant cancer compared with the intensity pre-DC chemotherapy ( $\mathrm{P}=0.054$; Table II). In addition, no significant difference in SCCA expression levels between patients with chemotherapy-sensitive and chemotherapy-resistant cancer was observed $(\mathrm{P}>0.05)$. The positive and negative immunohistochemical staining images for SCCA are presented in Fig. 1. 
Table III. Overall survival rate of chemotherapy-sensitive ( 2 complete response and 7 partial response) and chemotherapy-resistant (12 stable disease and 0 progressive disease) patients with cervical cancer following surgery.

\begin{tabular}{|c|c|c|c|}
\hline Factor & $\begin{array}{c}\text { Chemotherapy-sensitive, } \\
\text { n/total n (\%) }\end{array}$ & $\begin{array}{c}\text { Chemotherapy-resistant, } \\
\text { n/total n }(\%)\end{array}$ & $\begin{array}{l}\text { Total mortality } \\
\text { n/total n (\%) }\end{array}$ \\
\hline \multicolumn{4}{|c|}{ Mortality based on SCCA level pre-chemotherapy } \\
\hline+ & $2 / 7(28.6)$ & $4 / 10(40.0)$ & $6 / 17(35.3)$ \\
\hline- & $0 / 2(0.0)$ & $0 / 2(0.0)$ & $0 / 4(0.0)$ \\
\hline \multicolumn{4}{|c|}{ Mortality based on SCCA level post-chemotherapy } \\
\hline+ & $2 / 3(66.7)$ & $4 / 8(50.0)$ & $6 / 11(54.5)$ \\
\hline- & $0 / 6(0.0)$ & $0 / 4(0.0)$ & $0 / 10(0.0)$ \\
\hline Total mortality & $2 / 9(22.2)$ & 4/12 (33.3) & 6/21 (28.6) \\
\hline
\end{tabular}

SCCA, squamous cell carcinoma antigen; CR, complete response; PR, partial response; PD, progressive disease; SD, stable disease; +, SCCA-positive expression; -, SCCA-negative expression.
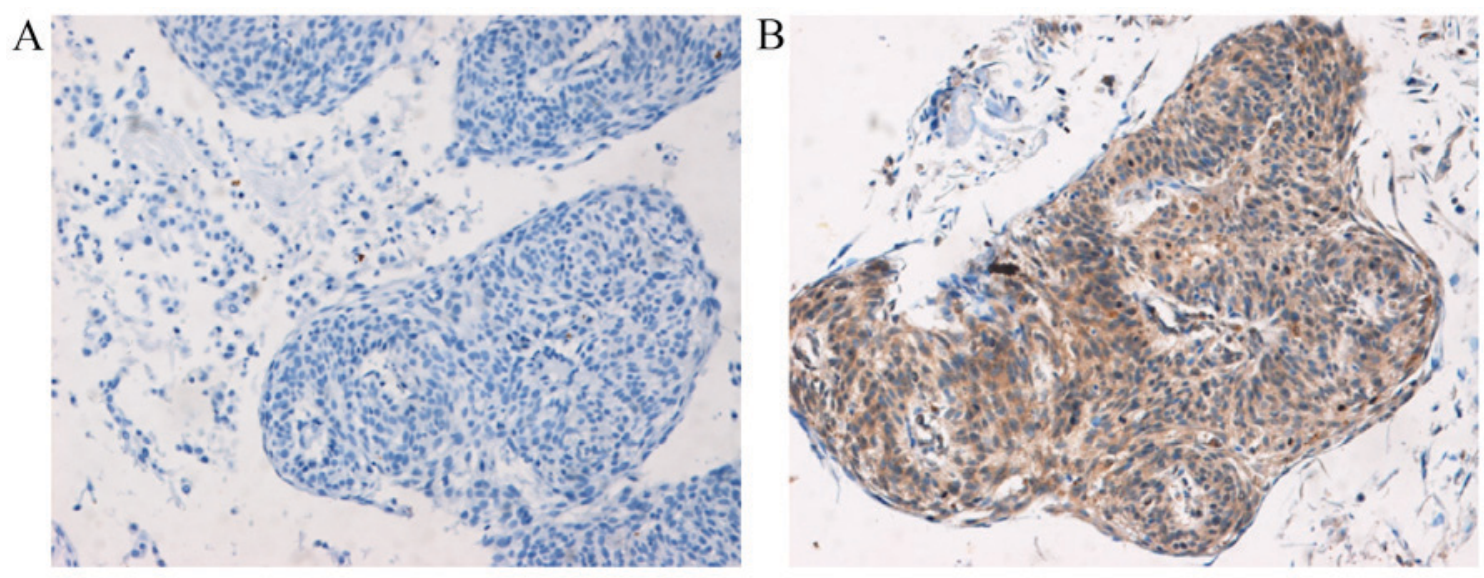

Figure 1. Representative images of immunohistochemical staining results for SCCA protein (magnification, x100). (A) SCCA-negative expression tissue with no staining. (B) SCCA-positive expression tissue exhibited brownish-yellow staining. SCCA, squamous cell carcinoma antigen.

Survival analysis. No patients were lost to follow-up. The mean follow-up duration was $37.9 \pm 12.6$ months. During the follow-up, 5 patients succumbed to disease recurrence and 1 patient succumbed due to other causes. Furthermore, no recurrence was observed in the remaining patients at the time of the final follow-up (December 2014). In addition, there were no cases of mortality among the enrolled patients with negative SCCA expression, prior to or following DC chemotherapy. By contrast, a mortality rate of 35.3\% (6/17) and 54.5\% (6/11) was observed in patients with positive SCCA expression prior to and following DC chemotherapy, respectively (Table III). Furthermore, the mortality rate of the chemotherapy-sensitive group $(22.2 \% ; 2 / 9)$ and chemotherapy-resistant $(33.3 \% ; 4 / 12)$ cancer were similar (Table III). No significant difference in the cumulative survival rate between patients with positive and negative SCCA expression prior to chemotherapy $(\mathrm{P}=0.211$; log-rank test; Fig. 2A) was identified; however, the survival curve based on chemotherapy sensitivity and SCCA expression levels following chemotherapy revealed significant differences between patients with positive expression and negative expression of SCCA ( $\mathrm{P}=0.009$, log-rank test; Fig. 2B). Furthermore, the cumulative survival rate between patients with chemotherapy-sensitive and chemotherapy-resistant cancer was similar ( $\mathrm{P}=0.698$, log-rank test; Fig. 2C).

\section{Discussion}

The present study demonstrated that DC chemotherapy may decrease the expression levels of the SCCA protein, which is concordant with the results of previous studies (21-23). The present results demonstrated that SCCA expression levels were significantly reduced by DC chemotherapy in patients with chemotherapy-sensitive cancer, but no significant difference was observed in the expression levels of SCCA prior to and following DC chemotherapy in patients with chemotherapy-resistant cancer. Furthermore, no statistically significant difference between chemotherapy-sensitive and chemotherapy-resistant cancer in the proportion of cases that exhibited positive SCCA expression levels following chemotherapy was observed; however, the visually higher proportion of patients with positive expression of SCCA post-chemotherapy in chemotherapy-resistant cancer cases demonstrated the association between SCCA expression levels and sensitivity to chemotherapy. 
A

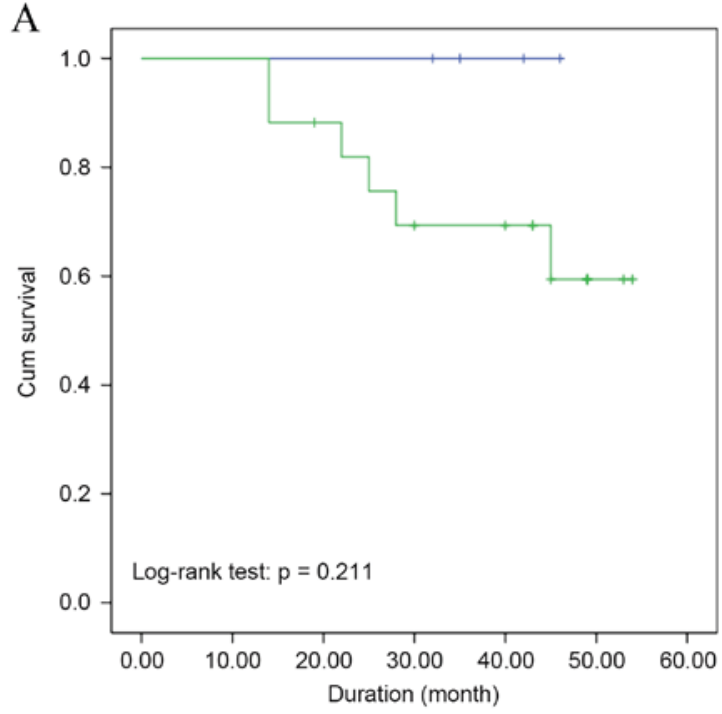

B

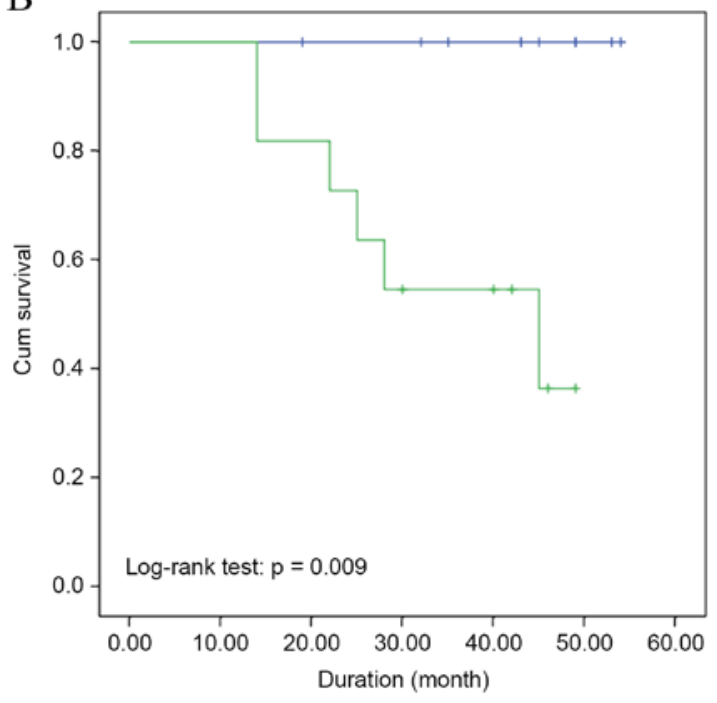

\section{C}

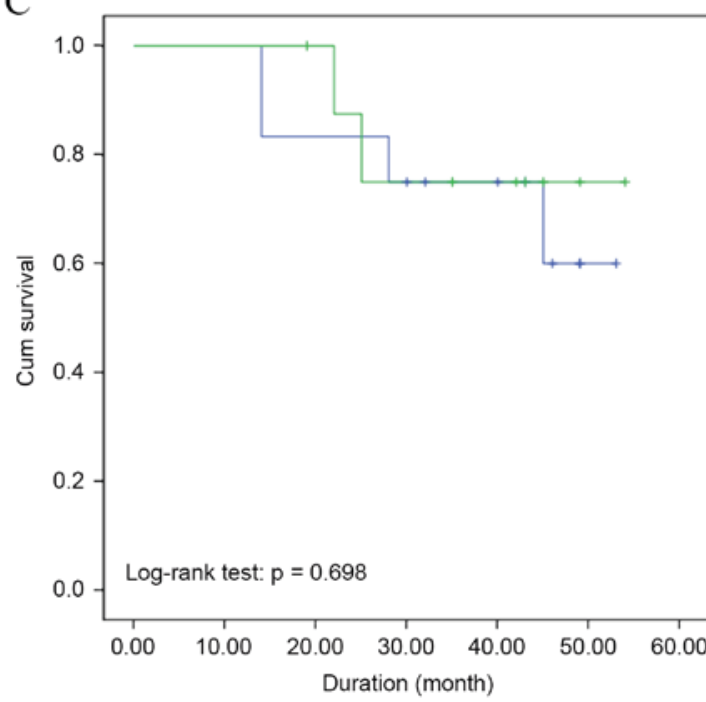

Expression of SCCA

before chemotherapy

$\neg$ Negative

- Negative-censored

+ Positive-censored
Expression of SCCA

after chemotherapy

$\neg$ Negative

Positive

+ Negative-censored

+ Positive-censored $\neg$ Chemotherapy-sensitive cancer

$\checkmark$ Chemotherapy-resistant cancer

+ Chemotherapy-sensitive cancer -censored

+ Chemotherapy-resistant cancer -censored

Figure 2. Survival curve based on the expression of SCCA (A) prior to and (B) following chemotherapy, and (C) chemotherapy sensitivity. No significant differences were observed in the cumulative survival rate between patients with positive and negative SCCA expression prior to chemotherapy (A; $\mathrm{P}=0.211)$. However, significant differences between these groups were identified following chemotherapy ( $\mathrm{B} ; \mathrm{P}=0.009)$. No significant difference was observed in the cumulative survival rate between patients with chemotherapy-sensitive and chemotherapy-resistant cancer ( $\mathrm{P}=0.698$; Fig. 2C). SCCA, squamous cell carcinoma antigen. 
A previous study identified SCCA as a serine protease inhibitor (27). In addition, the serine protease high temperature requirement factor A1 (HtrA1) was observed to mediate paclitaxel- and cisplatin-based chemotherapy resistance in ovarian and gastric cancer (28), whereas HtrA3 was proposed to be associated with the chemotherapy sensitivity of patients with lung cancer $(29,30)$. Therefore, the serine protease inhibitor activity of SCCA may be the primary mechanism underlying the association between SCCA expression levels and platinum-based chemotherapy sensitivity. SCCA is also a potent inhibitor of the cysteine proteinase cathepsin L (31). The chemotherapy efficacy in a mouse model of multistage cancer was able to be be enhanced when the cysteine cathepsin protease activity was inhibited (32). Therefore, the association between SCCA expression and chemotherapy sensitivity may also be due to the activity of SCCA as a cysteine proteinase inhibitor. The evidence for the role of SCCA in chemotherapy resistance is limited, and further studies will be required to investigate the underlying molecular mechanisms by which SCCA affects chemosensitivity in cervical cancer.

In the present study, no significant difference in SCCA expression was observed prior to chemotherapy between patients with chemotherapy-sensitive and chemotherapy-resistant cancer. This result was contrary to the findings of a previous study by Li et al (22), in which it was reported that elevated expression levels of SCCA were associated with the poor response of squamous cervical cancer to cisplatin-based NAC. The discrepancy in the data may be due to variations between cisplatin- and carboplatin-based NAC, as carboplatin has been observed to produce a higher overall response rate compared with cisplatin (33). Furthermore, there are differences in the mechanisms underlying the resistance to cisplatin and carboplatin (34). The small sample size used in the present study may be another reason for this discrepancy. Therefore, additional studies will be required to investigate the variations between cisplatin and carboplatin in association with SCCA expression levels.

In the present study, the results also revealed significant differences in survival between patients with SCCA-positive and -negative expression following chemotherapy. This result indicates the prognostic potential of SCCA for predicting the survival of patients with cervical patients who receive DC chemotherapy followed by radical surgery. However, no statistically significant difference between the survival of patients with positive and negative SCCA expression prior to chemotherapy was observed. A significant change in SCCA expression following chemotherapy was observed in patients with chemotherapy-sensitive cancer, but not in patients with chemotherapy-resistant cancer. Therefore, it may be hypothesized that the prognostic value of SCCA expression levels pre-DC chemotherapy are affected by the variable changes in SCCA expression post-DC chemotherapy, between patients with chemotherapy-sensitive and chemotherapy-resistant cancer. In addition, serum SCCA levels may also be affected by clinical stage, differentiation and lymph node metastasis (35). Further studies will be required to determine the association between chemotherapy sensitivity and SCCA expression levels, and to examine the effect of chemotherapy sensitivity on the prognostic role of SCCA in predicting the survival of patients with cervical cancer.
The primary limitation of the current study was the small sample size, which decreased the statistical power and affected the stability and reliability of the results. Another limitation was that docetaxel is not a drug frequently used in NAC, which may limit the wider clinical applications of the results. Further studies must be performed in order to elucidate the association between SCCA expression levels and the response to paclitaxel-based chemotherapy in cervical cancer, as this treatment was commonly used in the majority of previous studies that evaluated the use of a platinum-based regime. In addition, the absence of a correlation between antigen modification during chemotherapy and the modulation of the serum biomarker SCCA was important, as it may be one of the reasons for the change in SCCA expression levels following DC chemotherapy, and may have affected the results of the present study. Nonetheless, there are certain advantages of the results of the present study. Firstly, this study specifically considered DC chemotherapy and provided data demonstrating an association between SCCA expression levels and chemotherapy sensitivity. Secondly, it was observed that the association between SCCA expression levels and chemotherapy response may affect the prognostic value of pre-chemotherapy SCCA expression levels for cervical cancer survival. This suggests a novel target for further studies that may provide a broader basis for the clinical application of SCCA expression levels as an indicator of chemotherapy sensitivity, or as a prognostic factor for cervical cancer.

In conclusion, decreased SCCA expression levels following DC chemotherapy are associated with chemotherapy sensitivity. Therefore, SCCA expression levels post-DC chemotherapy have the potential to be used to evaluate the clinical prognosis following radical surgery for patients with cervical cancer; however, further studies with longer follow-up periods are required.

\section{Acknowledgements}

The present study was supported by The National Natural Science Foundation of China (grant no. 81270675) and The Shengjing Hospital of China Medical University (grant no. mc78).

\section{References}

1. Mombelli S, Kwiatkowski F, Abrial C, Wang-Lopez Q, de Boissieu P, Garbar C, Bensussan A and Curé H: Prognostic factors in operable breast cancer treated with neoadjuvant chemotherapy: Towards a quantification of residual disease. Oncology 88: 261-272, 2015.

2. Kwee RM and Kwee TC: Role of imaging in predicting response to neoadjuvant chemotherapy in gastric cancer. World J Gastroenterol 20: 1650-1656, 2014.

3. Rosenblatt R, Sherif A, Rintala E, Wahlqvist R, Ullén A, Nilsson S and Malmström PU; Nordic Urothelial Cancer Group: Pathologic downstaging is a surrogate marker for efficacy and increased survival following neoadjuvant chemotherapy and radical cystectomy for muscle-invasive urothelial bladder cancer. Eur Urol 61: 1229-1238, 2012.

4. Tanaka K, Miyata H, Yamasaki M, Sugimura K, Takahashi T, Kurokawa Y, Nakajima K, Takiguchi S, Mori M and Doki Y: Circulating miR-200c levels significantly predict response to chemotherapy and prognosis of patients undergoing neoadjuvant chemotherapy for esophageal cancer. Ann Surg Oncol 20 (Suppl 3): S607-S615, 2013 
5. Sardi JE, Boixadera MA and Sardi JJ: Neoadjuvant chemotherapy in cervical cancer: A new trend. Curr Opin Obstet Gynecol 17: 43-47, 2005.

6. Benedetti Panici P, Bellati F, Pastore M, Manci N, Musella A, Pauselli S, Angelucci M, Muzii L and Angioli R: An update in neoadjuvant chemotherapy in cervical cancer. Gynecol Oncol 107 (Suppl 1): S20-S22, 2007.

7. Shen SN, Wang LF, Jia YF, Hao YQ, Zhang L and Wang H: Upregulation of microRNA-224 is associated with aggressive progression and poor prognosis in human cervical cancer. Diagn Pathol 8: 69, 2013.

8. Punt S, Fleuren GJ, Kritikou E, Lubberts E, Trimbos JB, Jordanova ES and Gorter A: Angels and demons: Th17 cells represent a beneficial response, while neutrophil IL-17 is associated with poor prognosis in squamous cervical cancer. Oncoimmunology 4: e984539, 2015.

9. Jemal A, Bray F, Center MM, Ferlay J, Ward E and Forman D: Global cancer statistics. CA Cancer J Clin 61: 69-90, 2011.

10. Chen YJ, Shen MR, Chen YJ, Maa MC and Leu TH: Eps8 decreases chemosensitivity and affects survival of cervical cancer patients. Mol Cancer Ther 7: 1376-1385, 2008.

11. Watari H, Kanuma T, Ohta Y, Hassan MK, Mitamura T, Hosaka M, Minegishi T and Sakuragi N: Clusterin expression inversely correlates with chemosensitivity and predicts poor survival in patients with locally advanced cervical cancer treated with cisplatin-based neoadjuvant chemotherapy and radical hysterectomy. Pathol Oncol Res 16: 345-352, 2010.

12. Benedetti-Panici P, Greggi S, Colombo A, Amoroso M Smaniotto D, Giannarelli D, Amunni G, Raspagliesi F, Zola P, Mangioni $\mathrm{C}$ and Landoni F: Neoadjuvant chemotherapy and radical surgery versus exclusive radiotherapy in locally advanced squamous cell cervical cancer: Results from the Italian multicenter randomized study. J Clin Oncol 20: 179-188, 2002.

13. Kuo DY, Blank SV, Christos PJ, Kim M, Caputo TA, Pothuri B, Hershman D, Goldman N, Ivy PS, Runowicz CD, et al: Paclitaxel plus oxaliplatin for recurrent or metastatic cervical cancer: A New York Cancer Consortium Study. Gynecol Oncol 116 442-446, 2010

14. Köhler C, Oppelt P, Favero G, Morgenstern B, Runnebaum I, Tsunoda A, Schmittel A, Schneider A, Mueller M and Marnitz S: How much platinum passes the placental barrier? analysis of platinum applications in 21 patients with cervical cancer during pregnancy. Am J Obstet Gynecol 213: 206.e1-5, 2015.

15. Lorusso D, Petrelli F, Coinu A, Raspagliesi F and Barni S: A systematic review comparing cisplatin and carboplatin plus paclitaxel-based chemotherapy for recurrent or metastatic cervical cancer. Gynecol Oncol 133: 117-123, 2014.

16. Vacca A, Ribatti D, Iurlaro M, Merchionne F, Nico B, Ria R and Dammacco F: Docetaxel versus paclitaxel for antiangiogenesis. J Hematother Stem Cell Res 11: 103-118, 2002.

17. Rose PG, Blessing JA, Ball HG, Hoffman J, Warshal D, DeGeest K and Moore DH: A phase II study of docetaxel in paclitaxel-resistant ovarian and peritoneal carcinoma: A gynecologic oncology group study. Gynecol Oncol 88: 130-135, 2003.

18. Verschraegen CF, Sittisomwong T, Kudelka AP, Guedes Ed, Steger M, Nelson-Taylor T, Vincent M, Rogers R, Atkinson EN and Kavanagh JJ: Docetaxel for patients with paclitaxel-resistant Müllerian carcinoma. J Clin Oncol 18: 2733-2739, 2000.

19. Takekida S, Fujiwara K, Nagao S, Yamaguchi S, Yoshida N, Kitada F, Kigawa J, Terakawa N and Nishimura R: Phase II study of combination chemotherapy with docetaxel and carboplatin for locally advanced or recurrent cervical cancer. Int J Gynecol Cancer 20: 1563-1568, 2010.

20. Wei H, Wang N, Zhang Y, Wang S, Pang X, Zhang J, Luo Q, Su Y and Zhang S: Clinical significance of Wnt-11 and squamous cell carcinoma antigen expression in cervical cancer. Med Oncol 31: 933, 2014.
21. Jeong BK, Huh SJ, Choi DH, Park W, Bae DS and Kim BG: Prognostic value of different patterns of squamous cell carcinoma antigen level for the recurrent cervical cancer. Cancer Res Treat 45: 48-54, 2013

22. Li X, Zhou J, Huang K, Tang F, Zhou H, Wang S, Jia Y, Sun H, Ma D and Li S: The predictive value of serum squamous cell carcinoma antigen in patients with cervical cancer who receive neoadjuvant chemotherapy followed by radical surgery: A single-institute study. PLoS One 10: e0122361, 2015.

23. Yin M, Hou Y, Zhang T, Cui C, Zhou X, Sun F, Li H, Li X, Zheng J, Chen X, et al: Evaluation of chemotherapy response with serum squamous cell carcinoma antigen level in cervical cancer patients: A prospective cohort study. PLoS One 8: e54969, 2013.

24. Gómez R, Ossa CA, Montoya ME, Echeverri C, Ángel G, Ascuntar J, Borrero M, Gil M, Herrera S, Gutiérrez E, et al: Impact of immunohistochemistry-based molecular subtype on chemosensitivity and survival in Hispanic breast cancer patients following neoadjuvant chemotherapy. Ecancermedicalscience 9: 562,2015 .

25. Kim HS and Song YS: International federation of gynecology and obstetrics (FIGO) staging system revised: What should be considered critically for gynecologic cancer? J Gynecol Oncol 20: 135-136, 2009.

26. van Persijn van Meerten EL, Gelderblom H and Bloem JL: RECIST revised: Implications for the radiologist. A review article on the modified RECIST guideline. Eur Radiol 20: 1456-1467, 2010

27. Suminami Y, Kishi F, Sekiguchi K and Kato H: Squamous cell carcinoma antigen is a new member of the serine protease inhibitors. Biochem Biophys Res Commun 181: 51-58, 1991.

28. Chien J, Aletti G, Baldi A, Catalano V, Muretto P, Keeney GL, Kalli KR, Staub J, Ehrmann M, Cliby WA, et al: Serine protease HtrA1 modulates chemotherapy-induced cytotoxicity. J Clin Invest 116: 1994-2004, 2006.

29. Thompson D, Chien J, Staub J, Molina J and Shridhar V: Loss of HtrA3, a serine protease involved in smoking-related lung cancer: Implications for chemoresistance. Clin Cancer Res 12: B70, 2006.

30. Clausen T, Southan C and Ehrmann M: The HtrA family of proteases: Implications for protein composition and cell fate. Mol Cell 10: 443-455, 2002.

31. Takeda A, Yamamoto T, Nakamura Y, Takahashi T and Hibino T: Squamous cell carcinoma antigen is a potent inhibitor of cysteine proteinase cathepsin L. FEBS Lett 359: 78-80, 1995

32. Bell-McGuinn KM, Garfall AL, Bogyo M, Hanahan D and Joyce JA: Inhibition of cysteine cathepsin protease activity enhances chemotherapy regimens by decreasing tumor growth and invasiveness in a mouse model of multistage cancer. Cancer Res 67: 7378-7385, 2007.

33. Moore KN, Herzog TJ, Lewin S, Giuntoli RL, Armstrong DK, Rocconi RP, Spannuth WA and Gold MA: A comparison of cisplatin/paclitaxel and carboplatin/paclitaxel in stage IVB, recurrent or persistent cervical cancer. Gynecol Oncol 105: 299-303, 2007

34. Stewart DJ: Mechanisms of resistance to cisplatin and carboplatin. Crit Rev Oncol Hematol 63: 12-31, 2007.

35. Hong JH, Tsai CS, Chang JT, Wang CC, Lai CH, Lee SP, Tseng CJ, Chang TC and Tang SG: The prognostic significance of pre- and posttreatment SCC levels in patients with squamous cell carcinoma of the cervix treated by radiotherapy. Int J Radiat Oncol Biol Phys 41: 823-830, 1998. 